\title{
Ru(II) Complexes of Tetradentate Ligands Related to 2,9-Di(pyrid-2'-yl)-1,10-phenanthroline
}

\author{
Gang Zhang, Ruifa Zong, Huan-Wei Tseng and Randolph P. Thummel* \\ Department of Chemistry, 136 Fleming Building, University of Houston, \\ Houston, TX 77204-5003
}

\section{Supporting Information}

\section{Table of Contents}

Table S1. ${ }^{1} \mathrm{H}$ NMR data for tetradentate ligands and their $\mathrm{Ru}(\mathrm{II})$ complexes ${ }^{*}$ 
Table S1. ${ }^{1} \mathrm{H}$ NMR data for tetradentate ligands and their Ru(II) complexes ${ }^{\star}$

\begin{tabular}{|c|c|c|c|c|c|c|c|c|c|c|}
\hline \multirow{2}{*}{ Ligand/Complex } & \multicolumn{10}{|c|}{ Aromatic Protons } \\
\hline & $\mathrm{H} 3$ & $\mathrm{H} 4$ & H5 & H6 & H3' & $\mathrm{H} 4^{\prime}$ & $\mathrm{H}^{\prime}$ & H6' & $\mathrm{H} 7^{\prime}$ & $\mathrm{H} 8$ \\
\hline $1 \mathbf{a}$ & 8.93 & 8.45 & 7.89 & -- & 8.82 & 7.47 & 8.07 & 9.12 & -- & -- \\
\hline 1b & 8.90 & 8.43 & 7.89 & -- & 9.02 & -- & 7.28 & 8.66 & -- & -- \\
\hline 1c & 8.82 & 8.38 & 7.84 & -- & 8.20 & -- & 6.63 & 8.44 & -- & -- \\
\hline 1d & 8.84 & 8.43 & 7.89 & -- & 9.50 & -- & 7.96 & 8.91 & -- & -- \\
\hline 3 & 8.57 & 8.30 & 7.79 & -- & -- & 7.09 & 7.24 & -- & -- & -- \\
\hline 4 & 8.82 & 8.44 & 7.92 & -- & -- & 7.90 & 7.40 & -- & -- & -- \\
\hline $5 \mathbf{a}$ & 9.36 & 8.52 & 7.94 & -- & 9.20 & 8.49 & 7.96 & 7.63 & 7.80 & 8.27 \\
\hline $5 \mathbf{b}$ & 9.39 & 8.53 & 7.93 & -- & 9.22 & 8.50 & 8.34 & 7.57 & 9.13 & \\
\hline 6 & 9.02 & 9.10 & -- & -- & 8.84 & 7.48 & 8.08 & 9.79 & -- & -- \\
\hline$\left[\mathrm{Ru}(\mathbf{1 a})(\mathbf{2} \mathbf{b})_{2}\right]\left(\mathrm{PF}_{6}\right)_{2}$ & \multicolumn{2}{|c|}{$8.89,8.81$} & 8.60 & -- & 8.69 & 8.38 & 8.16 & 10.29 & -- & -- \\
\hline$\left[\mathrm{Ru}(\mathbf{1 b})(\mathbf{2} \mathbf{b})_{2}\right]\left(\mathrm{PF}_{6}\right)_{2}$ & \multicolumn{2}{|c|}{$8.84,8.77$} & 8.57 & -- & 8.54 & -- & 7.96 & 10.05 & -- & -- \\
\hline$\left[\mathrm{Ru}(\mathbf{1 c})(\mathbf{2 b})_{2}\right]\left(\mathrm{PF}_{6}\right)_{2}$ & \multicolumn{2}{|c|}{$8.86,8.66$} & 8.50 & -- & 7.81 & -- & 7.14 & 9.55 & -- & -- \\
\hline$\left[\mathrm{Ru}(\mathbf{3})(\mathbf{2 b})_{2}\right]\left(\mathrm{PF}_{6}\right)_{2}$ & \multicolumn{2}{|c|}{$8.63,8.61$} & 8.45 & -- & -- & 8.34 & 7.81 & -- & -- & -- \\
\hline$\left[\mathrm{Ru}(\mathbf{4})(\mathbf{2 b})_{2}\right]\left(\mathrm{PF}_{6}\right)_{2}$ & \multicolumn{2}{|c|}{$9.11,8.82$} & 8.62 & -- & -- & 9.06 & \multicolumn{2}{|c|}{$7.96,7.83$} & 8.05 & -- \\
\hline$\left[\mathrm{Ru}(\mathbf{5 a})(\mathbf{2 b})_{2}\right]\left(\mathrm{PF}_{6}\right)_{2}$ & \multicolumn{2}{|c|}{$9.20,9.08$} & 8.54 & -- & 8.86 & 9.20 & 8.42 & 7.93 & 8.04 & 9.27 \\
\hline$\left[\mathrm{Ru}(\mathbf{5 b})(\mathbf{2} \mathbf{b})_{2}\right]\left(\mathrm{PF}_{6}\right)_{2}$ & \multicolumn{2}{|c|}{$9.18,8.92$} & 8.71 & -- & 8.92 & 9.07 & 8.83 & 8.20 & 9.99 & \\
\hline$\left[\mathrm{Ru}(\mathbf{6})(\mathbf{2 b})_{2}\right]\left(\mathrm{PF}_{6}\right)_{2}$ & \multicolumn{2}{|c|}{$9.55,9.07$} & 8.61 & 8.29 & 8.76 & 8.42 & 8.18 & 10.32 & -- & -- \\
\hline
\end{tabular}

* Measured at room temperature. Ligand spectra were recorded in $\mathrm{CDCl}_{3}$ and reported in ppm referenced to $\mathrm{CHCl}_{3}$ at $7.26 \mathrm{ppm}$. Complex spectra were recorded in acetone- $d_{6}$ and reported in ppm referenced to acetone at $2.04 \mathrm{ppm}$. 
Table S2. Data collection and processing parameters for $\left[\mathrm{Ru}(\mathbf{3})(\mathbf{2 b})_{2}\right]\left(\mathrm{PF}_{6}\right)_{2}$

\begin{tabular}{|c|c|}
\hline Compound & {$\left[\mathrm{Ru}(\mathbf{3})(\mathbf{2} \mathbf{b})_{2}\right]\left(\mathrm{PF}_{6}\right)_{2}$} \\
\hline Molecular formula & $\mathrm{C}_{32} \mathrm{H}_{30} \mathrm{~F}_{12} \mathrm{~N}_{8} \mathrm{P}_{2} \mathrm{Ru}$ \\
\hline Formula weight & 917.65 \\
\hline Space group & $\mathrm{P} \overline{1} \quad$ (triclinic) \\
\hline \multirow[t]{6}{*}{ Cell constants } & $\mathrm{a}=10.3341(9) \AA$ \\
\hline & $\mathrm{b}=13.6041(12)$ \\
\hline & $c=14.0934(13)$ \\
\hline & $\alpha=86.131(1)^{\circ}$ \\
\hline & $\beta=72.135$ (1) \\
\hline & $\gamma=70.607$ \\
\hline Formula units per cell & $Z=2$ \\
\hline Volume & $1777.5 \AA^{3}$ \\
\hline Density & $\rho=1.715 \mathrm{~g} / \mathrm{cm}^{3}$ \\
\hline Absorption coefficient & $\mu=6.33 \mathrm{~cm}^{-1}$ \\
\hline Temperature & $\mathrm{T}=223(2) \mathrm{K}$ \\
\hline Radiation (Mo K $\alpha$ ) & $\lambda=0.71073 \AA$ \\
\hline Collection range & $3^{\circ} \leq 2 \theta \leq 48^{\circ}$ \\
\hline Total data collected & 8447 \\
\hline Independent data & 5625 \\
\hline Total variables & 421 \\
\hline R1 & 0.0541 with $\mathrm{I}>4 \sigma(\mathrm{I})$ \\
\hline wR & 0.1395 \\
\hline
\end{tabular}


$\mathrm{X}$-ray crystallographic files for $\left[\mathrm{Ru}(\mathbf{3})(\mathbf{2} \mathbf{b})_{2}\right]\left(\mathrm{PF}_{6}\right)_{2}$ (in CIF format). The material is available free of charge via the Internet at http://pubs.acs.org.

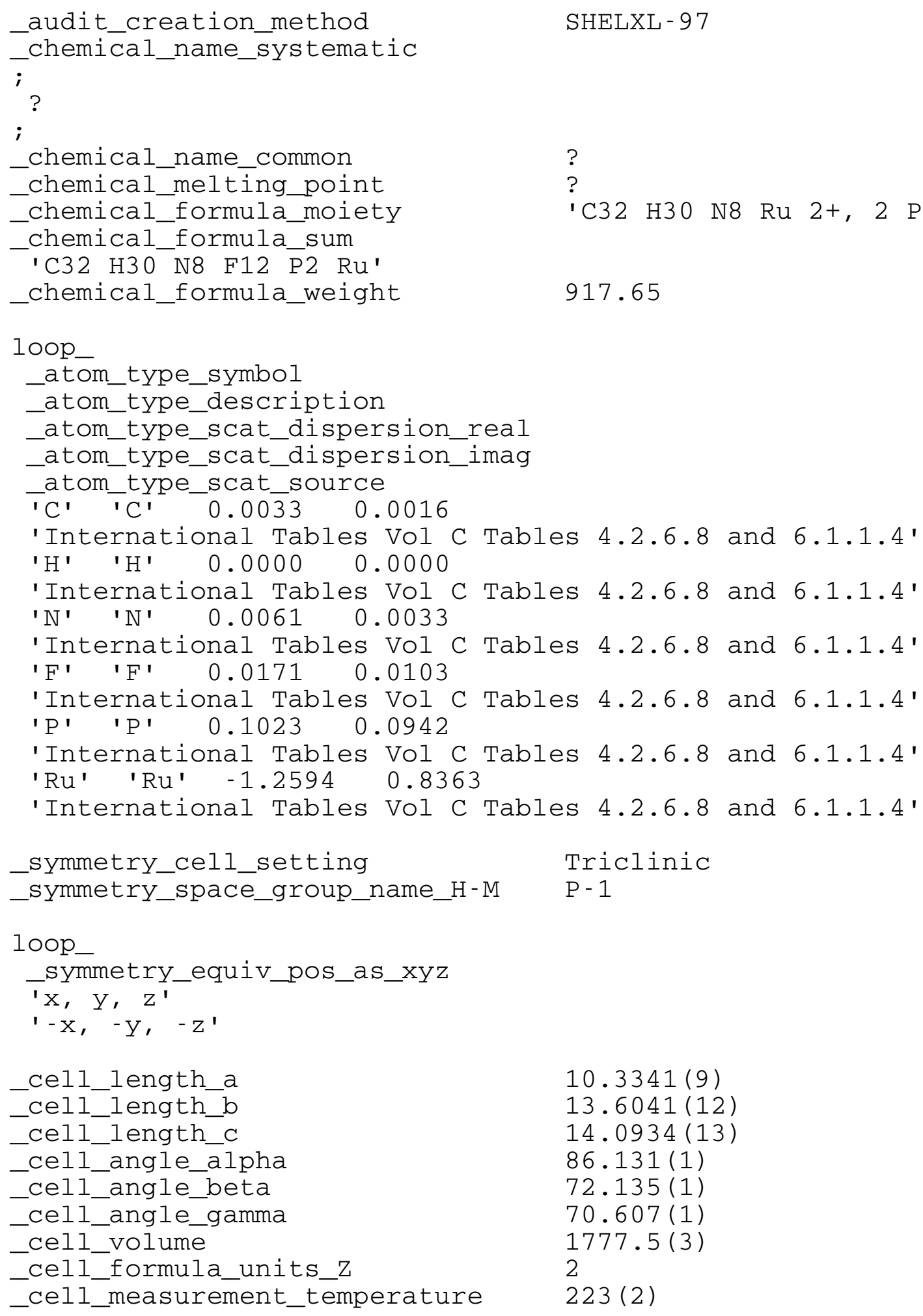

_cell_measurement_temperature $223(2)$

$10.3341(9)$

$13.6041(12)$

$14.0934(13)$

$86.131(1)$

$72.135(1)$

$70.607(1)$

$1777.5(3)$

2 
_cell_measurement_reflns_used

_cel1_measurement_theta_min

_cell_measurement_theta_max

_expt1_crystal_description

_expt1_crystal_colour

_expt1_crystal_size_max

_expt1_crystal_size_mid

_expt1_crystal_size_min

_expt1_crystal_density_diffrn

_expt1_crystal_density_method

_expt1_crystal_F_000

_expt1_absorpt_coefficient_mu

_expt1_absorpt_correction_type

_expt1_absorpt_correction_T_min

_expt1_absorpt_correction_T_max

_expt1_absorpt_process_details
3480

1.52

24.17

'Diamond column'

'Dark carmine'

0.25

0.10

0.06

1.715

' not measured'

920

0.633

Empirical

0.8123

0.9555

?

_publ_section_experimental

;

All measurements were made with a Siemens SMART platform diffractometer equipped with a 4K CCD APEX II detector. A hemisphere of data (1271 frames at $6 \mathrm{~cm}$ detector distance) was collected using a narrow-frame algorithm with scan widths of $0.30 \backslash \%$ in omega and an exposure time of $30 \mathrm{~s} /$ frame. The data were integrated using the Bruker-Nonius SAINT program, with the intensities corrected for Lorentz factor, polarization, air absorption, and absorption due to variation in the path length through the detector faceplate. A psi scan absorption correction was applied based on the entire data set. Redundant reflections were averaged. Final cell constants were refined using 3480 reflections having I>10\S(I), and these, along with other information pertinent to data collection and refinement, are listed in Table 1. The Laue symmetry was determined to be -1 , and the space group was shown to be either P1 or P-1. Both independent anions were found to be disordered, and this was treated by refinement of ideal rigid bodies at each of the major orientations, having occupancy factors based on comparison of their average isotropic displacement parameters. ;

publ_section_figure_captions

;

Fig. 1 -- View of the cation showing the atom numbering scheme for

key atoms. Thermal ellipsoids are 40\% equiprobability envelopes, with hydrogens omitted.

Fig. 2 -- Isotropic view of the cation.

Fig. 3 -- Top view of the cation with axial ligands removed, showing 
the atom numbering for the main ligand. Thermal ellipsoids are

$40 \%$ equiprobability envelopes.

Fig. 4 -- Side view of the cation.

Fig. 5 -- Packing of the molecules in the unit cell. Only one orientation of each disordered anion is shown at any site.

;

_diffrn_ambient_temperature

_diffrn_radiation_wavelength

_diffrn_radiation_type

_diffrn_radiation_source

_diffrn_radiation_monochromator

_diffrn_measurement_device_type

_diffrn_measurement_method

_diffrn_detector_area_resol_mean

_diffrn_standards_decay_o

_diffrn_reflns_number

_diffrn_reflns_av_R_equivalents

_diffrn_reflns_av_sigmaI/netI

_diffrn_reflns_limit_h_min

_diffrn_reflns_limit_h_max

_diffrn_reflns_limit_k_min

_diffrn_reflns_limit_k_max

_diffrn_reflns_limit_l_min

_diffrn_reflns_limit_l_max

_diffrn_reflns_theta_min

_diffrn_reflns_theta_max

_reflns_number_total

_reflns_number_gt

_reflns_threshold_expression

_computing_data_collection

2005)'

_computing_cell_refinement

$2004) '$

_computing_data_reduction

2004)'

_computing_structure_solution

1997)'

_computing_structure_refinement 1997)'

_computing_molecular_graphics

_computing_publication_material
$223(2)$

0.71073

MoK\a

'fine-focus sealed tube'

graphite

'Bruker APEX II CCD'

$\mathrm{CCD}$

$4 \mathrm{~K}$

0.0

8447

0.0558

0.0536

$-11$

11

$-15$

15

0

16

1.52

24.17

5625

3894

$>$ 4sigma (I)

'APEX2 v1.0-27 (Bruker-Nonius,

'SAINT V7.12A (Bruker-Nonius,

' SAINT v7.12A (Bruker-Nonius,

'SHELXS V5.10 (G.M. Sheldrick,

'SHELXL V5.10 (G.M. Sheldrick,

?

?

_refine_special_details

;

Refinement of $\mathrm{F}^{\wedge} 2 \wedge$ against $\mathrm{ALL}$ reflections. The weighted $\mathrm{R}$ factor wR and goodness of fit $S$ are based on $F^{\wedge} 2 \wedge$, conventional $R$-factors $R$ are based 
on $F$, with $F$ set to zero for negative $F \wedge 2 \wedge$. The threshold expression of $F^{\wedge} 2 \wedge>4$ sigma $\left(F^{\wedge} 2 \wedge\right)$ is used only for calculating R-factors(gt) etc. and is not relevant to the choice of reflections for refinement. $\mathrm{R}$ factors based on $\mathrm{F}^{\wedge} 2 \wedge$ are statistically about twice as large as those based on $\mathrm{F}$, and $\mathrm{R}$ factors based on ALL data will be even larger. ;

_refine_ls_structure_factor_coef Fsqd refine_ls_matrix_type full _refine_ls_weighting_scheme calc _refine_ls_weighting_details

' $\operatorname{calc} \bar{w}=1 /[\backslash \mathrm{s} \wedge 2 \wedge(\mathrm{F} \circ \wedge 2 \wedge)+(0.0739 \mathrm{P}) \wedge 2 \wedge+8.4741 \mathrm{P}]$ where $\mathrm{P}=\left(\mathrm{F} \circ{ }^{\wedge} 2 \wedge+2 \mathrm{FC} \mathrm{C}^{\wedge} 2 \wedge\right) / 3^{\prime}$

_atom_sites_solution_primary direct

_atom_sites_solution_secondary

_atom_sites_solution_hydrogens

_refine_ls_hydrogen_treatment

_refine_ls_extinction_method

_refine_ls_number_reflns

refine_ls_number_parameters

_refine_ls_number_restraints

_refine_ls_R_factor_all

_refine_ls_R_factor_gt

_refine_ls_wR_factor_ref

refine_ls_wR_factor_gt

refine_ls_goodness_of_fit_ref

refine_ls_restrained_s_al $\overline{1}$

_refine_ls_shift/su_max

difmap

geom

mixed

none

5619

421

0

0.0761

0.0541

0.1608

0.1395

1.024

1.024

0.519

_refine_ls_shift/su_mean

0.028

loop

_atom_site_label

_atom_site_type_symbol

_atom_site_fract_x

_atom_site_fract_y

_atom_site_fract_z

-atom_site_u_iso_or_equiv

-atom_site_adp_type

_atom_site_occupancy

_atom_site_symmetry_multiplicity

_atom_site_calc_flag

_atom_site_refinement_flags

_atom_site_disorder_assembly

_atom_site_disorder_group

$\mathrm{Ru} \operatorname{Ru} 0.75676(5) \quad 0.74423(4) \quad 0.24792(3) \quad 0.02227(17) \quad U a n i 11$. .

N1 N $0.7346(5) \quad 0.8816(4) \quad 0.3252(3) \quad 0.0279$ (11) Uani 11 d . . .

C2 C $0.8083(7) \quad 0.9266(5) \quad 0.3644(5) \quad 0.0366(16)$ Uani 11 d . .

H2 $\mathrm{H} \quad 0.9037 \quad 0.8951 \quad 0.3656 \quad 0.044$ Uiso 11 calc $\mathrm{R}$. .

C3 C $0.7215(8) \quad 1.0243(5) \quad 0.4015$ (5) 0.0410 (17) Uani 11 d . . 
H3 $\mathrm{H} \quad 0.74621 .07180 .43210 .049$ Uiso 11 calc $\mathrm{R}$

$\mathrm{N} 4 \mathrm{~N} \quad 0.5925(6) \quad 1.0402(4) \quad 0.3862(4) \quad 0.0376(13)$

C5 C $0.6012(7) \quad 0.9529(4) \quad 0.3400(4) \quad 0.0284(14)$

$\begin{array}{lllllll}\text { C6 C } & 0.4944(6) & 0.9275(5) & 0.3076(4) & 0.0293(14)\end{array}$

C7 C $0.3469(7) \quad 0.9783(5) \quad 0.3200(5) \quad 0.0409(17)$

H7 H $0.3000 \quad 1.04410 .3530 \quad 0.049$ Uiso 11 calc

C8 C $0.2723(7) \quad 0.9318(6) \quad 0.2841(5) \quad 0.0419(17)$ Uani 11 d . .

H8 H $0.1740 \quad 0.96650 .2928 \quad 0.050$ Uiso 11 calc $R$.

C9 C $0.3378(7) \quad 0.8344(5) \quad 0.2347(5) \quad 0.0367(16)$ Uani 11 d . .

C10 C $0.2741(7) \quad 0.7731(6) \quad 0.1953(5) \quad 0.0435(18)$ Uani 11 d . .

$\mathrm{H} 10 \mathrm{H} \quad 0.17580 .8008 \quad 0.20040 .052$ Uiso 11 calc $\mathrm{R}$.

C11 C $0.3515(7) \quad 0.6761(6) \quad 0.1507(5) \quad 0.0400(17)$ Uani 11 d . .

$\mathrm{H} 11 \mathrm{H} \quad 0.30490 .6404 \quad 0.1250 \quad 0.048$ Uiso 11 calc $\mathrm{R}$.

C12 C $0.5009(7) \quad 0.6270(5) \quad 0.1419(4) \quad 0.0333(15)$ Uani 11 d . .

C13 C $0.5897(8) \quad 0.5268(5) \quad 0.1052$ (5) 0.0380 (16) Uani 11 d . .

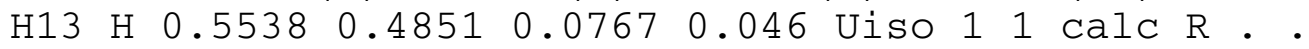

C14 C $0.7289(8) \quad 0.4873(5) \quad 0.1097(5) \quad 0.0385(16)$ Uani 11 d . .

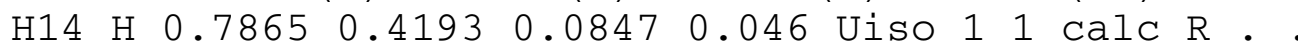

C15 C $0.7854(7) \quad 0.5492(5) \quad 0.1519(4) \quad 0.0316(14)$ Uani 11 d . .

C16 C $0.9194(7) \quad 0.5277(5) \quad 0.1762(4) \quad 0.0311(14)$ Uani 11 d . .

N17 N $1.0280(6) \quad 0.4381(4) \quad 0.1716(4) \quad 0.0395(14)$ Uani 11 d . .

C18 C $1.1219(7) \quad 0.4574(5) \quad 0.2113(6) \quad 0.0452(18)$ Uani 11 d . .

$\mathrm{H} 18 \mathrm{H} 1.2076 \quad 0.4090 \quad 0.21810 .054$ Uiso 11 calc $\mathrm{R}$.

C19 C $1.0677(6) \quad 0.5610(5) \quad 0.2396$ (5) 0.0362 (15) Uani 11 d . .

$\mathrm{H} 19 \mathrm{H} 1.1106 \quad 0.5961 \quad 0.2694 \quad 0.043$ Uiso $1 \quad 1$ calc $\mathrm{R}$.

$\mathrm{N} 20 \mathrm{~N} \quad 0.9412(5) \quad 0.6054(4) \quad 0.2175(4) \quad 0.0284(11) \quad \operatorname{Uani} 11 \mathrm{~d}$.

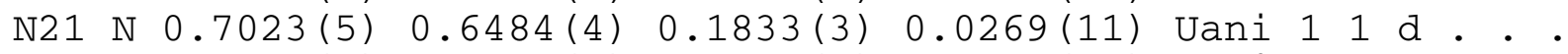

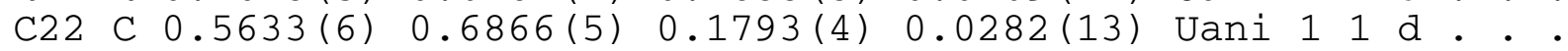

C23 C $0.4846(6) \quad 0.7883(5) \quad 0.2231(4) \quad 0.0272$ (13) Uani 11 d . .

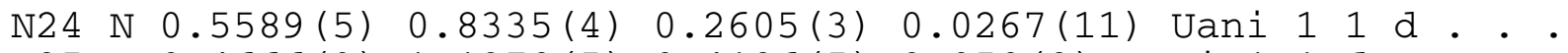

C25 C $0.4666(9) \quad 1.1370(5) \quad 0.4126(5) \quad 0.050(2)$ Uani 11 d . . .

$\mathrm{H} 25 \mathrm{~A} \mathrm{H} \quad 0.38631 .12210 .46110 .075$ Uiso 1 1 calc $\mathrm{R}$. .

H25B H 0.44001 .16320 .35310 .075 Uiso 11 calc $\mathrm{R}$.

$\mathrm{H} 25 \mathrm{C} \mathrm{H} 0.49101 .1890 \quad 0.44080 .075$ Uiso 1 1 calc R .

C26 C $1.0399(9) \quad 0.3345(5) \quad 0.1390(7) \quad 0.060(2)$ Uani 11 d . .

H26A H 1.12420 .28300 .15030 .091 Uiso 11 calc $\mathrm{R}$.

H26B H 1.04850 .33420 .06850 .091 Uiso 11 calc $R$. .

$\mathrm{H} 26 \mathrm{C}$ H $0.9546 \quad 0.3177 \quad 0.1767 \quad 0.091$ Uiso 11 calc $\mathrm{R}$. .

N27 N $0.8445(5) \quad 0.8078(4) \quad 0.1134(3) \quad 0.0258$ (11) Uani 11 d . .

C28 C $0.9873(6) \quad 0.7894(5) \quad 0.0799(5) \quad 0.0344$ (15) Uani 11 d . .

$\mathrm{H} 28 \mathrm{H} 1.04440 .74590 .11670 .041$ Uiso 11 calc $\mathrm{R}$. .

C29 C $1.0545(7) \quad 0.8303(6)-0.0045(5) \quad 0.0400(16)$ Uani 11 . .

H29 H $1.1550 \quad 0.8144-0.02410 .048$ Uiso 1 calc $R$

C30 C $0.9749(7) \quad 0.8952(5) \quad-0.0616(5) \quad 0.0343(15) \quad U a n i 11$ d . .

C31 C $0.8289(7) \quad 0.9144(5) \quad-0.0282(5) \quad 0.0382(16)$ Uani 11 d . .

H31 H $0.77040 .9575 \quad-0.06440 .046$ Uiso 1 calc $\mathrm{R}$. .

C32 C $0.7667(7) \quad 0.8707(5) \quad 0.0583(5) \quad 0.0338$ (15) Uani 11 d . .

H32 H 0.66640 .88560 .07930 .041 Uiso 11 calc $\mathrm{R}$. .

C33 C $1.0459(8) 0.9380(6)-0.1565(5) \quad 0.0487(19)$ Uani 11 d .

H33A H 0.9791 1.0031 -0.16900 .073 Uiso 11 calc $R$.

H33B H $1.07420 .8883-0.21130 .073$ Uiso 11 calc $R$.

H33C H $1.13040 .9502-0.15050 .073$ Uiso 11 calc R . .

N34 N $0.6918(5) \quad 0.6806(4) \quad 0.3864(3) \quad 0.0243(11) \quad \operatorname{Uani} 11 \mathrm{~d}$.

C35 C $0.7799(6) \quad 0.6558(5) \quad 0.4443(4) \quad 0.0311(14)$ Uani 11 d . . 


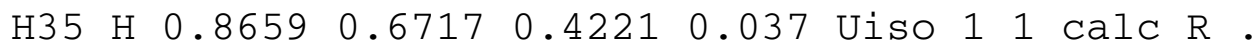

C36 C $0.7508(7) \quad 0.6087(5) \quad 0.5332$ (5) $0.0377(16)$ Uani 11 d . . H36 H $0.8164 \quad 0.5927 \quad 0.5703 \quad 0.045$ Uiso 11 calc $\mathrm{R}$.

C37 C $0.6243(7) \quad 0.5845(5) \quad 0.5689(5) \quad 0.0357(15)$ Uani 11 d . .

C38 C $0.5309(7) \quad 0.6126(5) \quad 0.5113(5) \quad 0.0323$ (14) Uani 11 d . .

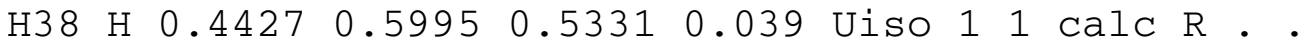

C39 C $0.5681(6) 0.6596(5) \quad 0.4221(4) \quad 0.0301(14)$ Uani 11 d . .

H39 H $0.50340 .67790 .3843 \quad 0.036$ Uiso 11 calc R .

C40 C $0.5884(9) \quad 0.5312(7) \quad 0.6655$ (5) $0.057(2) \quad \operatorname{Uani} 11$. . .

H40A H 0.49170 .52810 .68090 .085 Uiso 1 calc $\mathrm{R}$. .

H40B H $0.5940 \quad 0.5700 \quad 0.7186 \quad 0.085$ Uiso 11 calc $\mathrm{R}$.

$\mathrm{H} 40 \mathrm{C} \mathrm{H} 0.6563 \quad 0.4610 \quad 0.6596 \quad 0.085$ Uiso 1 calc R .

P1 P $0.5041(4) \quad 0.8088(3) \quad 0.8898(3) \quad 0.0437(5)$ Uiso 0.501 d PG A 1

F1 F $0.4000(6) \quad 0.8594(6) \quad 0.8240(5) \quad 0.116(6)$ Uiso 0.501 d PG A 1

F2 F $0.6083(6) \quad 0.7582(5) \quad 0.9557(4) \quad 0.070(3)$ Uiso 0.501 d PG A 1

F3 F $0.6353(6) \quad 0.7676(5) \quad 0.7912(3) \quad 0.053(2)$ Uiso 0.501 d PG A 1

F4 F $0.3730(5) \quad 0.8500(5) \quad 0.9885(4) \quad 0.051(2)$ Uiso 0.501 d PG A 1

F5 F $0.4689(8) \quad 0.7034(4) \quad 0.8914(5) \quad 0.108(4)$ Uiso 0.501 d PG A 1

F6 F $0.5394(8) \quad 0.9143(4) \quad 0.8883(5) \quad 0.097(4)$ Uiso 0.501 d PG A 1 P1' P $0.5107(4) 0.7972(3) \quad 0.8873(3) 0.0437(5)$ Uiso 0.501 d PG B 2

F1' F $0.4054(6) 0.8809(4) \quad 0.8369(5) \quad 0.047(2)$ Uiso 0.501 d PG B 2 F2' F $0.6160(6) 0.7134(4) \quad 0.9377(4) \quad 0.061(3)$ Uiso 0.501 d PG B 2

F3' F $0.5947(6) 0.7351(4) \quad 0.7829(3) \quad 0.050(2)$ Uiso 0.501 d PG B 2

F4' F $0.4266(7) \quad 0.8593(5) \quad 0.9916(4) \quad 0.084(3)$ Uiso 0.501 d PG B 2

F5' F $0.4052(6) 0.7300(5) \quad 0.9126(5) \quad 0.089(4)$ Uiso 0.501 d PG B 2

F6' F $0.6162(7) \quad 0.8643(5) \quad 0.8620(5) \quad 0.106(4)$ Uiso 0.501 d PG B 2

P2 P $0.9130(6) \quad 0.2455(4) \quad 0.4644(4) \quad 0.0531(8)$ Uiso $0.40 \quad 1$ d PG C 1

F7 F $0.9435(12) \quad 0.2555(9) \quad 0.5666(5) \quad 0.097(2)$ Uiso 0.40 d d PG C 1

F8 F $0.8824(11) \quad 0.2355(8) \quad 0.3622(5) \quad 0.097(2)$ Uiso $0.40 \quad 1$ d PG C 1 F9 F $0.7685(8) \quad 0.2230(8) \quad 0.5241(7) \quad 0.097(2)$ Uiso 0.401 d PG C 1 F10 F 1.0574(8) $0.2679(8) \quad 0.4046(7) \quad 0.097(2)$ Uiso 0.401 d PG C 1 F11 F $0.9994(10) \quad 0.1240(5) \quad 0.4572(8) \quad 0.097(2)$ Uiso 0.401 d PG C 1 F12 F $0.8265(10) \quad 0.3670(4) \quad 0.4716(8) \quad 0.097(2)$ Uiso 0.401 d PG C 1 P2' P $0.9302(6) 0.2369(4) 0.4617(4) 0.0531$ (8) Uiso 0.401 d PG D 2 F7' F $0.9077(11) \quad 0.2893(8) \quad 0.5656$ (5) 0.089 (2) Uiso 0.401 d PG D 2 F8' F $0.9527(10) 0.1846(7) \quad 0.3578(5) \quad 0.089(2)$ Uiso 0.401 d PG D 2

F9' F $0.7634(6) \quad 0.2511(8) \quad 0.4932(7) \quad 0.089(2)$ Uiso 0.401 d PG D 2 F10' F 1.0970(6) $0.2228(7) 0.4302(7) 0.089(2)$ Uiso 0.401 d PG D 2 F11' F $0.9609(11) 0.1254(5) \quad 0.5094(7) \quad 0.089(2)$ Uiso 0.401 d PG D 2 F12' F $0.8996(10) \quad 0.3484(5) \quad 0.4140(7) \quad 0.089(2)$ Uiso 0.401 d PG D 2 P2" P $0.9195(12) 0.2619(9) \quad 0.4424(8) \quad 0.0531(8)$ Uiso 0.201 d PG E 3 F7" F $0.825(2) \quad 0.3455(16) \quad 0.3846(17) 0.125(5)$ Uiso 0.201 d PG E 3 


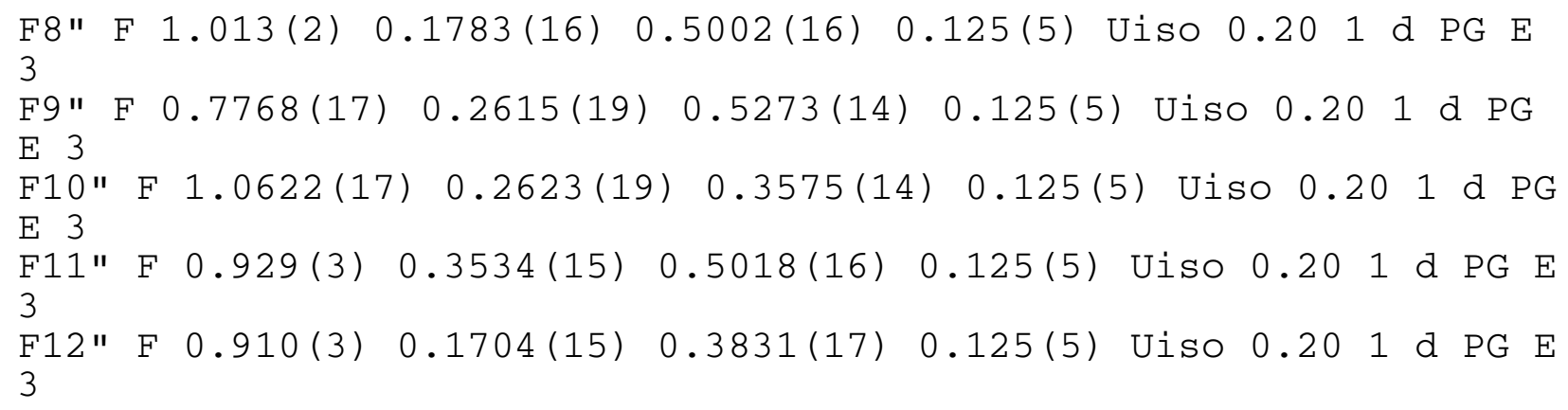




$\begin{array}{lllllll}\text { C33 } & 0.058(5) & 0.055(5) & 0.039(4) & 0.014(4) & -0.012(3) & -0.032(4) \\ \text { N34 } & 0.028(3) & 0.019(2) & 0.029(3) & 0.003(2) & -0.011(2) & -0.011(2) \\ \text { C35 } & 0.030(3) & 0.031(3) & 0.034(3) & 0.003(3) & -0.012(3) & -0.010(3) \\ \text { C36 } & 0.041(4) & 0.039(4) & 0.042(4) & 0.009(3) & -0.024(3) & -0.015(3) \\ \text { C37 } & 0.049(4) & 0.027(3) & 0.036(3) & 0.005(3) & -0.014(3) & -0.017(3) \\ \text { C38 } & 0.037(3) & 0.026(3) & 0.037(3) & 0.005(3) & -0.009(3) & -0.017(3) \\ \text { C39 } & 0.031(3) & 0.029(3) & 0.034(3) & 0.002(3) & -0.015(3) & -0.011(3) \\ \text { C40 } & 0.076(6) & 0.068(6) & 0.042(4) & 0.023(4) & -0.026(4) & -0.040(5)\end{array}$

_geom_special_details

;

All esds (except the esd in the dihedral angle between two $1 . s$. planes)

are estimated using the full covariance matrix. The cell esds are taken

into account individually in the estimation of esds in distances, angles

and torsion angles; correlations between esds in cell parameters are only

used when they are defined by crystal symmetry. An approximate (isotropic)

treatment of cell esds is used for estimating esds involving l.s. planes.

;

loop

_geom_bond_atom_site_label_1

-geom_bond_atom_site_label_2

_geom_bond_distance

_geom_bond_site_symmetry_2

_geom_bond_publ_flag

Ru N21 1.951(5) . ?

Ru N24 1.961(5) . ?

Ru N34 2.088(5) . ?

$\mathrm{Ru} N 27$ 2.099(5) . ?

Ru N1 2.138(5) . ?

$\mathrm{Ru} N 20$ 2.145(5) . ?

N1 C5 $1.359(8)$. ?

N1 C2 $1.369(8)$. ?

C2 C3 $1.363(10)$. ?

C3 N4 1.359(9) . ?

N4 C5 $1.357(8)$. ?

N4 C25 $1.483(8)$. ?

C5 C6 $1.459(9)$. ?

C6 N24 1.341(8) . ?

C6 C7 1.409(9) . ?

C7 C8 1.363(10) . ?

C8 C9 1.392(10) . ?

C9 $\mathrm{C} 231.397(8)$. ?

C9 C10 1.445(10) . ?

C10 C11 1.371(10) . ?

C11 C12 1.433(9) . ?

C12 C22 1.402(9). ?

C12 C13 1.394(9) . ? 


\begin{tabular}{|c|c|c|c|}
\hline $\mathrm{C} 13$ & C14 & $1.379(10$ & \\
\hline $\mathrm{C} 14$ & C15 & $1.418(9)$ & $\bullet$ \\
\hline 15 & N21 & $1.353(8)$ & \\
\hline 15 & C16 & $1.459(9)$ & \\
\hline 16 & N2 0 & $1.353(8)$ & \\
\hline 16 & N17 & $1.344(8)$ & \\
\hline 17 & C18 & $1.359(9)$ & \\
\hline & C2 6 & $1.467(9)$ & \\
\hline & C19 & $1.369(9)$ & \\
\hline & N2 0 & $1.367(8)$ & \\
\hline 1 & C22 & $1.374(7)$ & \\
\hline & C23 & $1.420(8)$ & \\
\hline 23 & N24 & $1.360(8)$ & \\
\hline 27 & C2 8 & $1.344(7)$ & \\
\hline 27 & C32 & $1.349(8)$ & \\
\hline 0 & $\mathrm{C} 29$ & $1.366(9)$ & \\
\hline 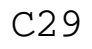 & C30 & $1.390(9)$ & \\
\hline & C31 & $1.373(9)$ & \\
\hline & C33 & $1.497(9)$ & \\
\hline & C32 & $1.389(9)$ & \\
\hline & C39 & $1.341(8)$ & \\
\hline & C35 & $1.350(7)$ & \\
\hline & C36 & $1.363(9)$ & \\
\hline & C37 & $1.390(9)$ & \\
\hline 37 & C38 & $1.390(9)$ & \\
\hline ד & $\mathrm{C} 40$ & $1.501(9)$ & \\
\hline C38 & C39 & $1.376(8)$ & \\
\hline 1 & F3 1 & $.5899 . ?$ & \\
\hline 1 & F6 1 & .5900 & \\
\hline P1 & F4 1 & .5900 & \\
\hline P1 & F1 1 & .5901 & \\
\hline $\mathrm{P} 1$ & F5 1 & .5901 & \\
\hline $\mathrm{P} 1$ & F2 1 & .5901. & \\
\hline P1' & F1' & 1.5899 & ? \\
\hline P1' & F5' & 1.5899 & ? \\
\hline P1' & F3' & 1.5900 & ? \\
\hline $1^{\prime}$ & $\mathrm{F} 2^{\prime}$ & 1.5900 & ? \\
\hline$P \perp$ & F4' & 1.5901 & ? \\
\hline P1' & $\mathrm{F} 6^{\prime}$ & 1.5901 & ? \\
\hline P2 & F8 1 & .589 & \\
\hline P2 & F12 & 1.5900 & \\
\hline P2 & F10 & 1.5900 & \\
\hline P2 & F9 1 & .59 & \\
\hline P2 & F11 & 1.5900 & \\
\hline P2 & F7 1 & .5901 . ? & \\
\hline $\mathrm{P} 2$ & F11 & ' 1.5899 & ? \\
\hline P2 & F10 & ' 1.5899 & ? \\
\hline P2' & F7' & 1.5900 & ? \\
\hline P2' & F8' & 1.5900 & ? \\
\hline P2 & F12 & ' 1.5900 & ? \\
\hline P2' & F9' & 1.5900 & ? \\
\hline P2" & F7" & 1.5900 & ? \\
\hline & F11 & " 1.5900 & ? \\
\hline & F9" & 1.5900 & \\
\hline
\end{tabular}




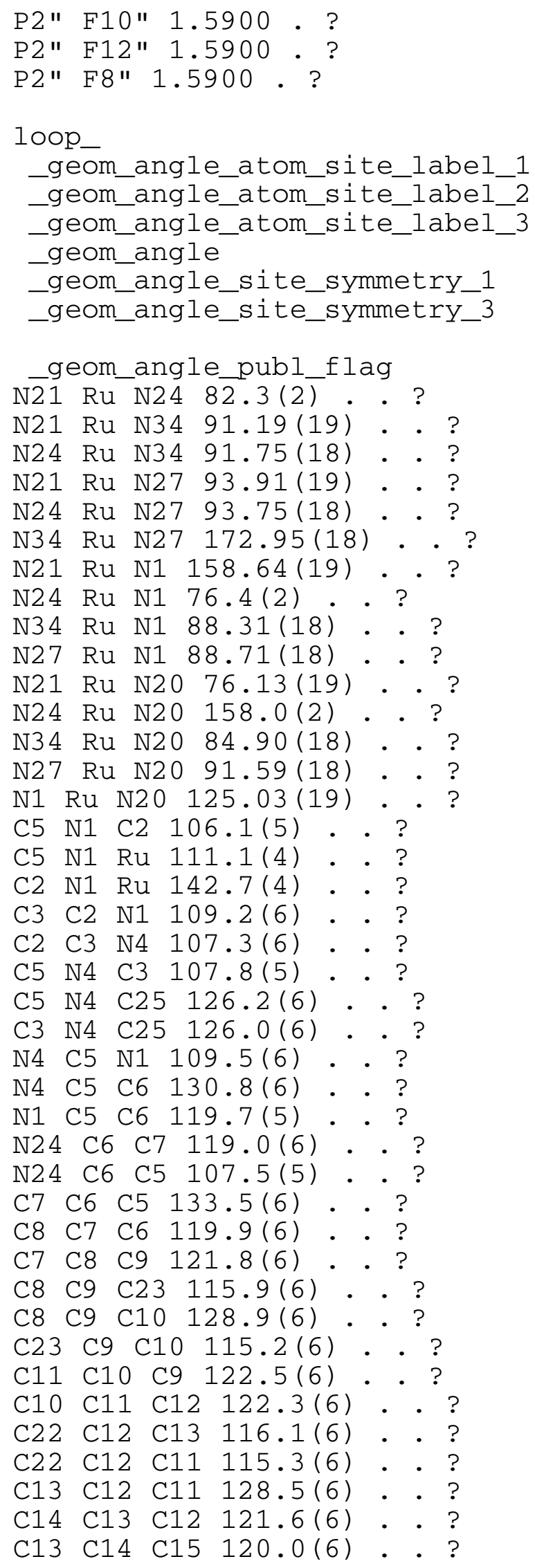




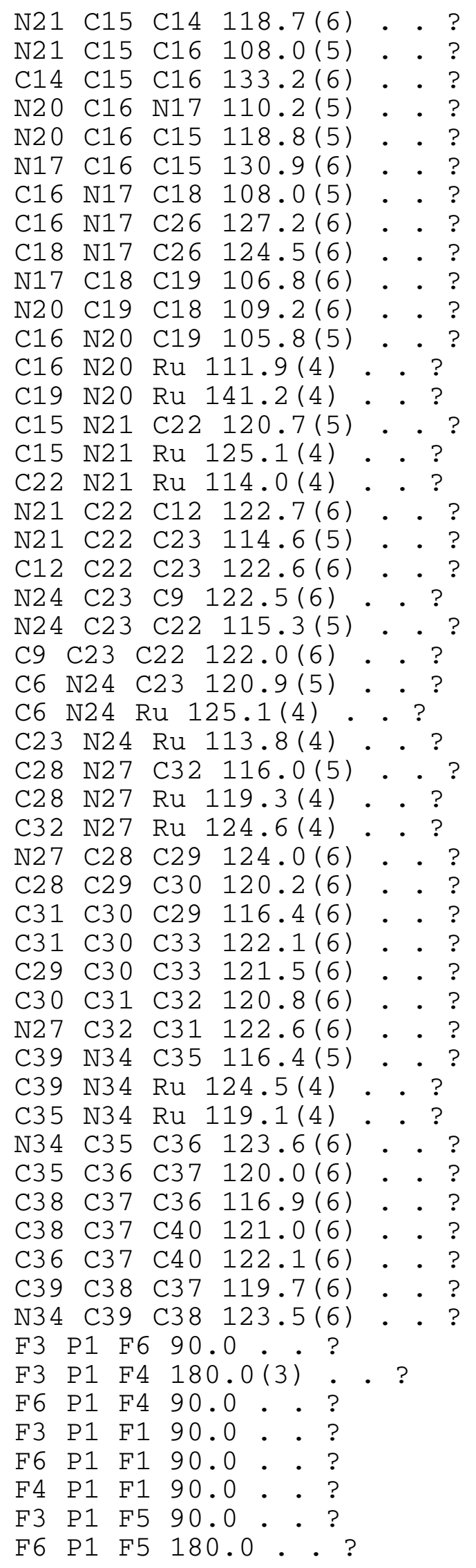




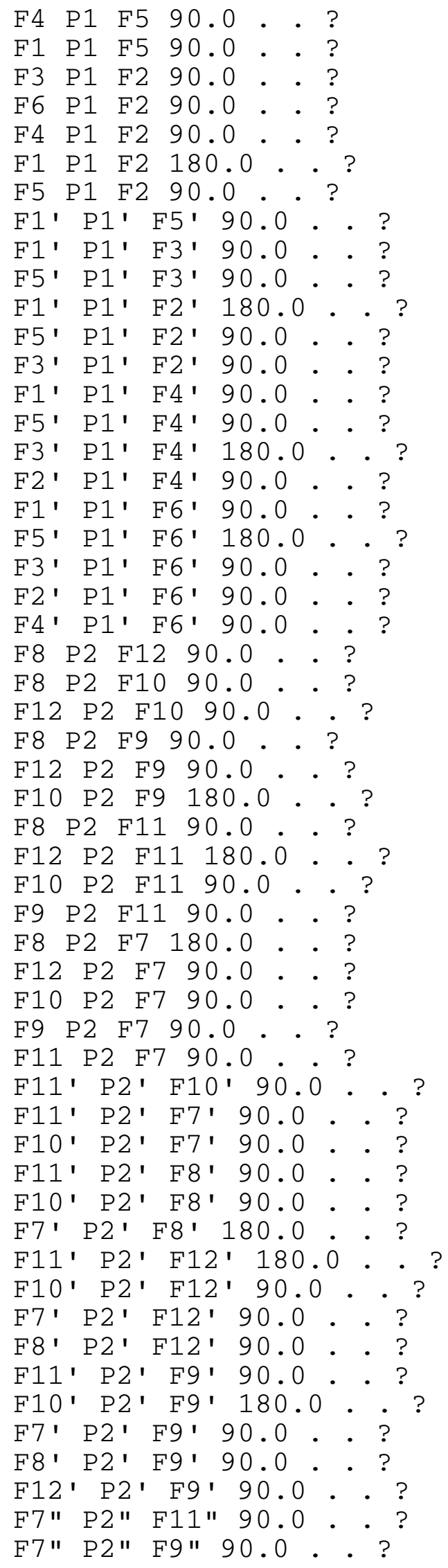




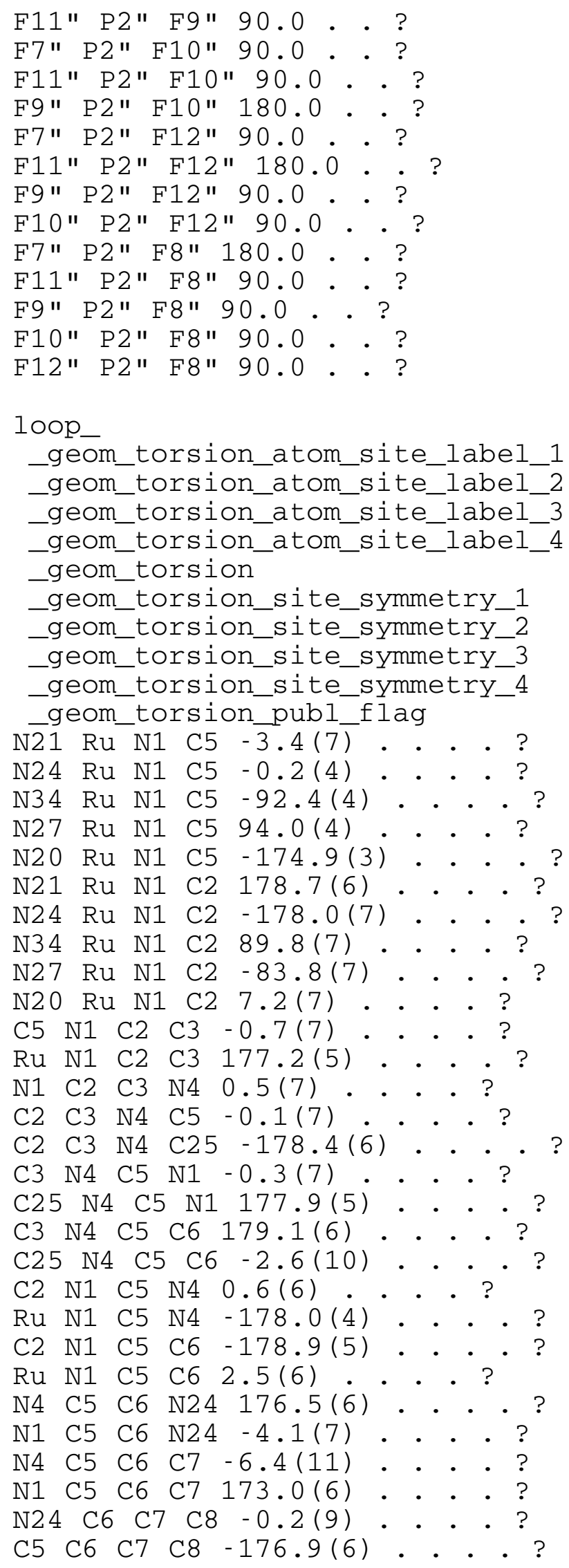




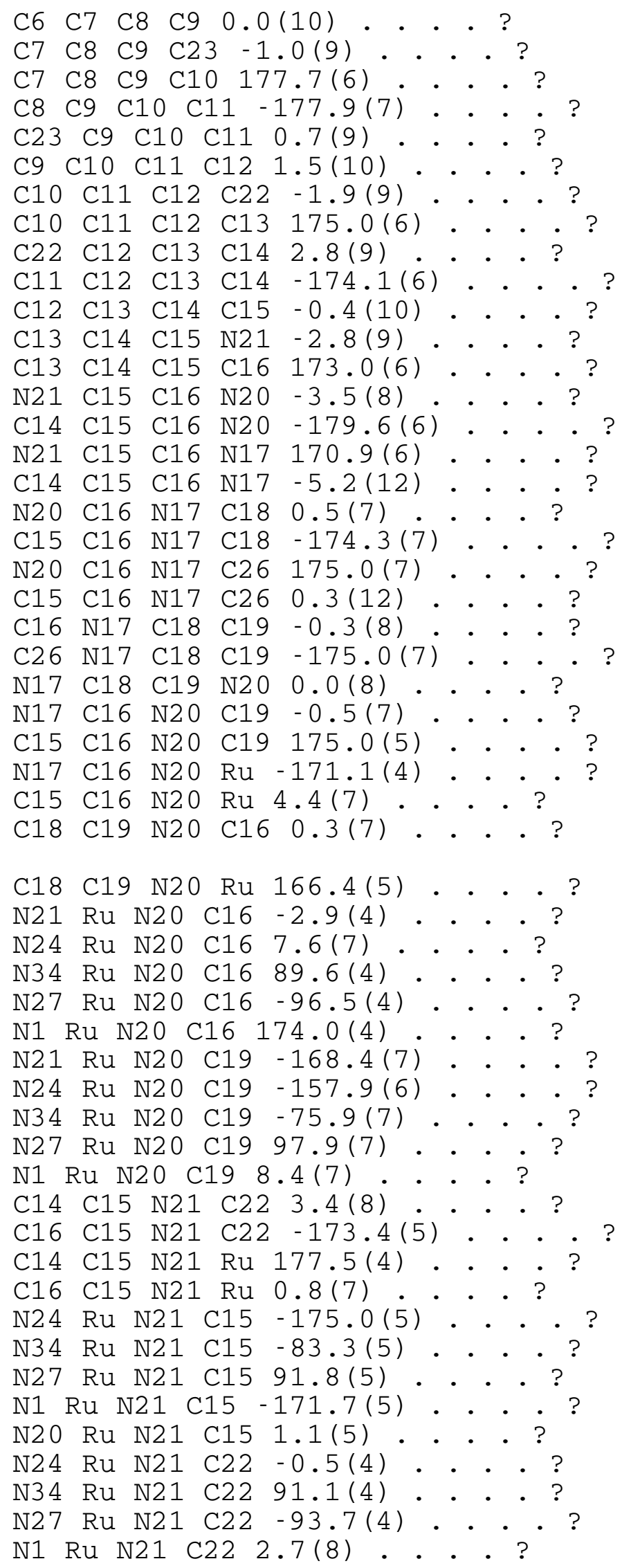




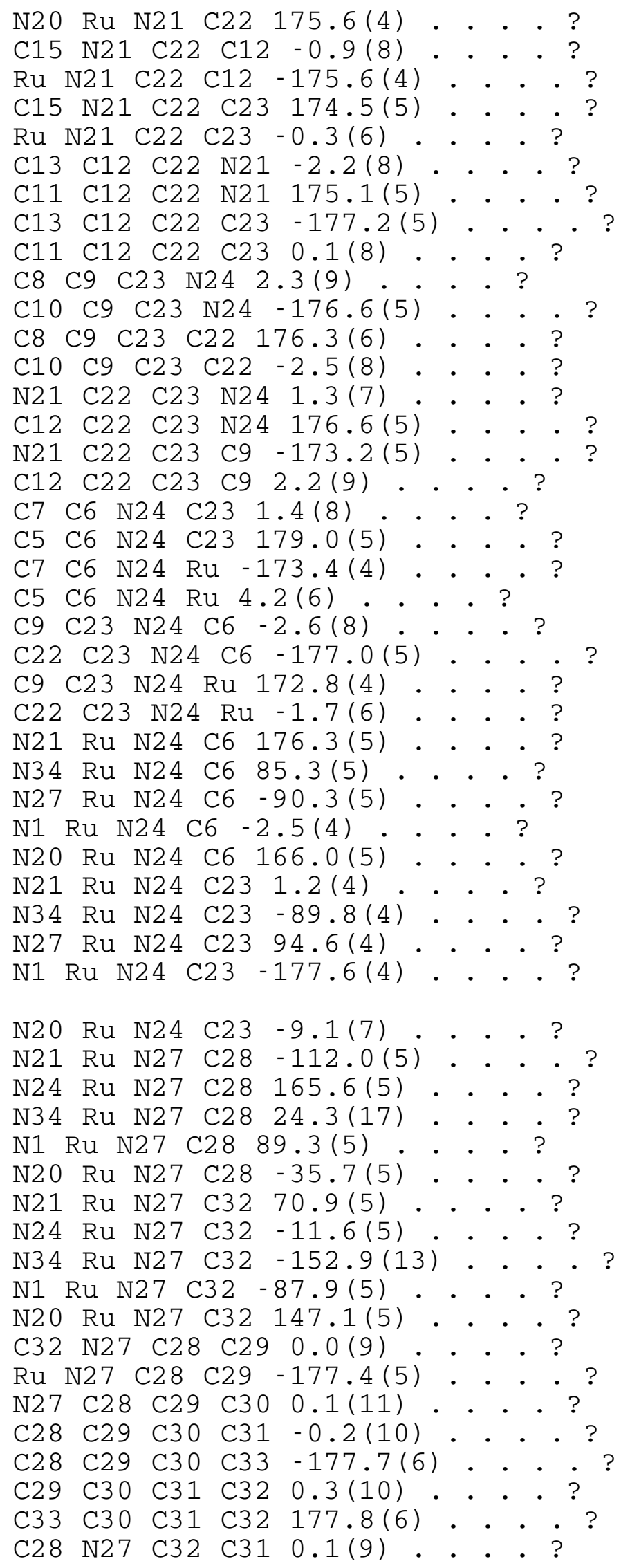




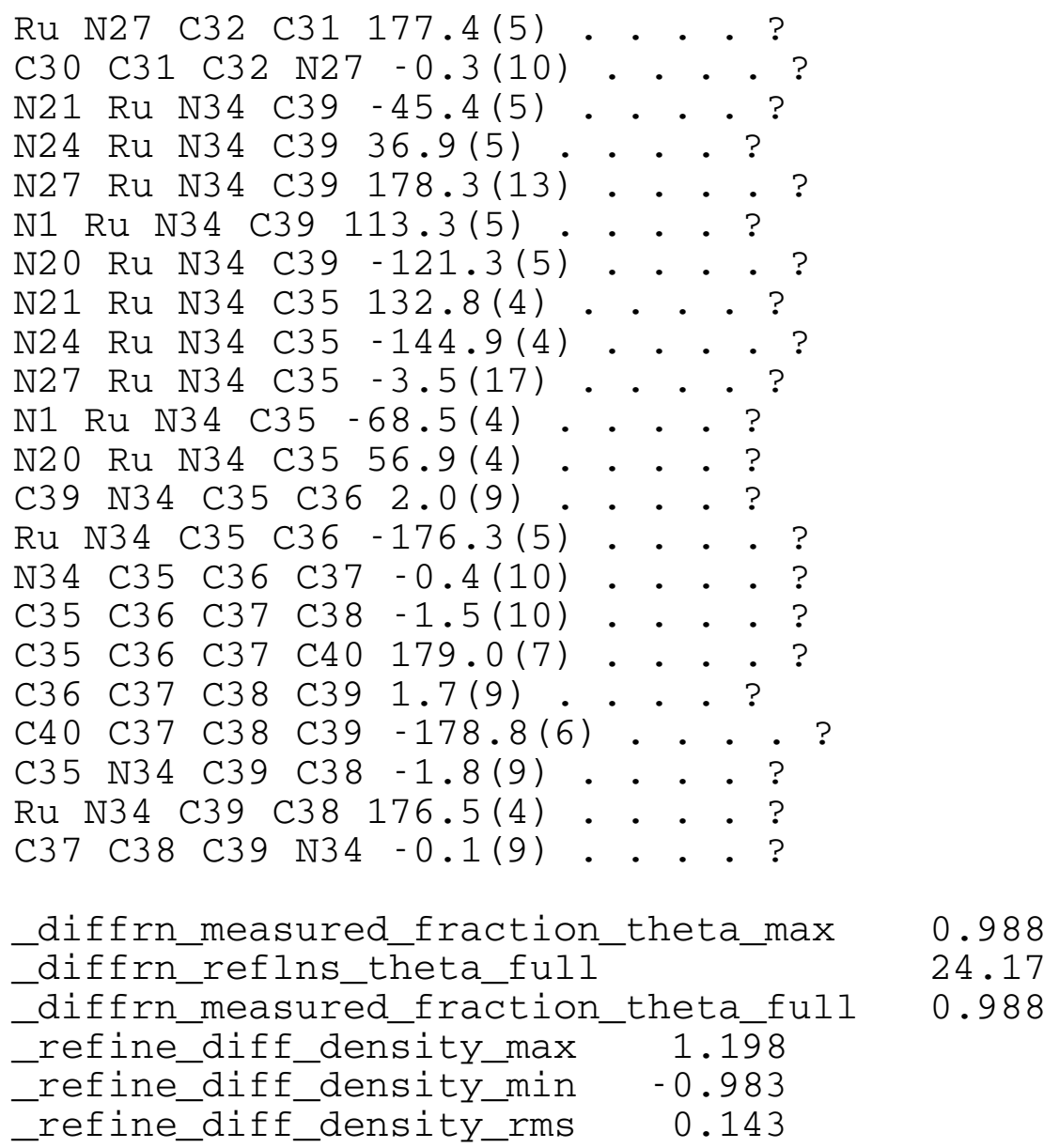

